\title{
Rapport sur l'éthique de la recherche dans les sciences du comportement ${ }^{(1)}$ humain
}

\author{
Rapporteurs : Anne Fagot-Largeault et Yves Laporte
}

En France, depuis la loi "sur la protection des personnes qui se prêtent à des recherches biomédicales " du 2) décembre 1988, les "essais ou expérimentations organisés et pratiqués sur l'être humain en vie du développement des commaissances biologiques ou médicales " se déroulent dans un cadre juridique précis et contraignant. Par contraste, le législateur n'a pas porté son attention sur la protection des persomnes qui se prêtent à des recherches comportementales, et les investigations réalisées sur l'être humain en v'ue du développement des comnaissances dans les disciplines qu'on nomme "sciences humaines" sont

(1) Il est admis dans tout ce qui suit que parlet est un compontement, et que la dénomination sciences du comportement humain n'exclut pas les disciplines climiques, telles que la psychologie d'inspiration anahtique. (etle demomination a l'aramtage de souligner qu'il s'agrit ici de recherche sue l'être humain autre que biomédicale.

(2) In Code de déntologie a été adopté on l 1961 (révisé en 1976) par la Société frangaise de P'sycho logie. II s'appliquait anx membres de la Société. Cie code a ele rejmis et actualise, is la suite de "lévolution de la propession et sa législation" (loi du 25 juiltet 1985) par litssociation mationale des organisations de psychologues (ANOP). (A Cide de déontologie des psychologues (1987) s'applique en thérie a tous les posychologrues frangais, et aux étudiants en psychologie.

II s'agit d'uil code de démtologie prosessionmelle,

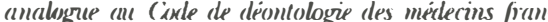
sais. Il ne s'agit pas d'un code d'éthique de la recherche comme l'est pour les médecins la déclaration a'Helsinki-Tokvo de l'Association médicale mondiale). (is code compente des reggles qui, siappli-

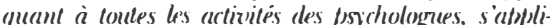
quent aussi anx activités de recherrhe. Règles gémérales: "Ne mellre en opurire que des mojens qui respectent la dignite humaine" (tot 2). "Livite de muire" (.trt. 15). Règles particulimes touchant le secret professiommel (.Arts $t$ i 12 )

$\mathrm{m} / \mathrm{s} n^{\circ} 12$ vol. 9, decemire 93 demeurées dans une situation juridique floue. Le Comité consultatif national d'éthique pour les sciences de la vie et de la santé (C.C.NE) récapitule ci-après: (1) les principes éthiques qui doivent guider toute investigation sur des sujets humains; (2) les problèmes particuliers qu’il a identifiés à propos de la recherche comportementale, et les éléments de solutions qu'il propose. Au terme de ce travail, le C.CNE invite tous les chercheurs en sciences humaines, les institutions scientifiques qui animent la recherche en sciences humaines, les autorités administratives compétentes, et le législateur, à une réflexion com- mune, et à une large concertation, en v'ue d'élaborer le cadre éthique et juridique dans lequel notre société souhaite que les investigations expérimentales sur les conduites humaines soient effectuées à l'avenir.

Dans son rapport Éthique et connaissance (1990), le C.CNE écrit: "Chercher à comnaitre scientifiquement l'être humain est un bien, mais cela ne peut se faire au prix de la justice, de la sécurité ou de l'autonomie des persomnes" (p. 74). Cela s'applique à la recherche sur l'homme, aussi bien dans les sciences du comportement que dans les sciences biomédicales. D’ailleurs,
7. Ie psychologrue est soumis à la reggle du secret pro. jessiommel définie iri comme secret psychologique. "s. (elle reggle doit s'appliquer alans des comditions analogues a celles qui somt défmies par liarticle 37.8 du ciode pénal.

"9. Ein particulier; il est rappele que ce secret doit soetendre, dans le domaine prive des persomnes, a tout ce que le psychologrue "a vu, entendu ou compris" an cours de sa pratique ou de ses recherches." " I1). Le secret doit être sancregarde aussi bien dams les paroles que dans la consernation et la diffusion des documents. Ie psychologue doit faire en sorte que les éléments issus de son travail (comptes rendus, conclusions, rapports, exposés, etc.) soient toujours rédigés, présentés et classés de maniere i préserver et ì consenver ce secret.

"Il. Dans sa coopération auec d'autres spécialistes, egalement soumis at secret projessiommel, le psychologue partage avec eux les infornations strictement nécessaires a la prise en charge en équipe d'un "client". "

"12. Il veille ì samuegarder le secret de lidentite des personnes lors de la constitution de fichiers de domnees, en conformiti aued la loi du 6avil 1978, relative a linformation et anx libertes.

"13. Ein dehors des cas d'obligation legale, le psychologue we peut être délie de som secret par quiconque même par ceux que ce secret concerme. Deux reggles concerment le traviail scientifique, far le biais du deroir de formation
"17. Tout psychologrue, quelle que soit sa spécialite, doit sünformer constamment des progries scienlificpues de sa discipline, el se former en conséguence. il tient compte dans son traviail de ces progrès et s'efforce diy concourir. Il accoptera toutes les reggles, exigences et contraintes qu'imposent les traviaux scienti/iques."

"19. Tout psychologue s'allache à rechercher et i appliquer des criteres el des méthodes scientifiquement contrölees et communicatizes, excluant de ce fait le principe al'autorite.

l'ne reggle peut s'appliquer ì la demande de consentement éclairé.

"18. Ie psychologue se garde de restreindre l'autonomie d'autrui el, en particulier, ses possibilités d'information, sa liberté de jusement el de decision. " Che regle spéciale vise le traitement des animaux d'expérience :

"26. Iarsque ses activités portent sur le comportement animal en vue de comprendre le comportement humain, il s'efforce d'assurer le bientêtre et la survie des animaux étudiés. "L'absence d'une règle équivalente visant le traitement des personnes se prêtant i la recherche comportementale implitue que les sujets de recherche ne sont pas traités differemment des autres sujets auxquels le psychologue a affaire dans son activité professiomelle (donc, par exemple, qu'un traitement diffétent en situation "thérapeutique" et "non thérapeutique" n'est pas entrisagé). 
dans le rapport de 1990 (ch. 2), le CCNE a pris en compte un ensemble de recherches à l'intersection du domaine biomédical et du domaine comportemental: études sur l'apprentissage, sur l'adaptation à l'environnement et aux tâches de travail (ergonomie), sur les réactions de l'organisme humain aux conditions extrêmes (hyperbarie, hypobarie, microgravité, climats extrêmes, déphasage des rythmes nycthéméraux, exploits sportifs de compétition ou d'endurance, civils ou militaires), sur la qualité de vie et les nuisances environnementales. La protection des personnes qui se prêtent à des recherches biomédicales dépend en France de la loi n 88-1138 du 20 décembre 1988 (modifiée $\left.n^{\circ} 90-86\right)$. La protection des personnes qui se prêtent à des recherches comportementales dépend pour le moment de la déontologie des chercheurs (exemple: Code de déontologie des psychologues (ㄴ) ).

Les recommandations que le C.C.NE va formuler sur l'éthique de la recherche comportementale doivent, par souci de cohérence, se situer en continuité avec la loi n $88-1138$ du 20 décembre 1988. Les grandes lignes des directives concernant la recherche sur l'homme qui ont été formulées aux États-Unis par la National Commission for the Protection of Human Subjects of Biomedical and Behavioral Research (1974-78), ou au Canada par le Conseil de recherches médicales CCRM (1986), puis par le National Council on Bioethics in Human Research (NCBHR), sont les mêmes pour l'ensemble de la recherche sur l'être humain, dans les sciences biomédicales et dans les sciences humaines, même si, secondairement, des problèmes spéciaux sont identifiés selon les disciplines (cancérologie, psychiatrie, ethnologie, etc.), ou pour différentes catégories de sujets étudiés (enfants, populations captives ou asilaires, personnes âgées en perte d'autonomie, etc.). On admettra donc que les grands principes éthiques qui régissent la recherche sur l'être humain américaine : principes de justice, de bienfaisance, de respect de l'autonomie des personnes), ainsi que les règles qui en découlent (règle d'équité ou de non-discrimination, règle de minimisation des risques et d'optimisation des bénéfices, règle du consentement) sont les mêmes, qu'il s'agisse de recherche biomédicale, ou de recherche comportementale. On admettra également que la procédure consistant à soumettre les protocoles de recherche sur l'homme, avant leur exécution, à l'examen d'un "comité indépendant " ou "comité d'éthique ", ou "comité de protection des personnes " est applicable à la recherche comportementale. Dans ce qui suit, on parlera de "CCPPRC " (Comités consultatifs de protection des personnes dans la recherche comportementale), sans préjuger leur rapport avec les CCPPRB (Comités consultatifs de protection des personnes dans la recherche biomédicale) institués par la loi n88-1138 du 20 décembre 1988 (qui sera envisagé plus loin).

\section{Grandes lignes}

\section{La liberté des personnes}

La liberté a un aspect négatif (indépendance) : ne pas être amené à faire ce qu'on ne veut pas, et un aspect positif (autonomie) : agir en accord avec ce qu'on veut réellement pour soi-même. On admet habituellement que respecter la liberté des personnes implique que l'on accepte la règle: aucune investigation ne sera conduite sur des personnes humaines sans que ces personnes aient donné à cette investigation leur consentement ${ }^{(3)}$ " libre, éclairé et exprès" (L. 209-9). En adaptant la loi $n^{\circ} 88-1138$ du 20 décembre 1988 (Art. L. 2909-9) : - le consentement est exprès s'il est "donné par écrit ou, en cas d'impossibilité, attesté par un tiers.. indépendant des investigateurs "; - le consentement est éclairé si le sujet a été suffisamment informé, s'il

(3) Sur la notion de consentement el ses ambiguilés, voir Thouvenin (1992). a compris l'information, s'il a eu un délai de réflexion avant de faire connaître son choix. Pour que l'information soit suffisante, la loi du 20 décembre 1988 précise que l'investigateur doit faire connaître au sujet: l'objectif de la recherche, sa méthodologie et sa durée; les bénéfices attendus, les contraintes et les risques prévisibles, y compris en cas d'arrêt de la recherche avant son terme; l'avis du (CCPPRC:;

- le consentement est libre si l'investigateur s'abstient de toute pression, coercition, ou incitation forte (argent, réussite à un examen, avantage de carrière, chantage affectif..). Par ailleurs, le consentement à la recherche est révocable. Les sujets doivent être informés qu'ils peuvent à tout moment cesser de participer, sans encourir de sanction ni de reproche.

\section{La sécurité. Le coût humain}

Dans la recherche biomédicale, on admet que certains protocoles de recherche impliquent pour les personnes qui s'y prêtent un «bénéfice individuel direct” (Art. L. 209-1). Cette notion de bénéfice (potentiel) pour le sujet sert en médecine à autoriser l'inclusion dans des protocoles de recherche, à certaines conditions (Art. L. 209-6), des personnes dont le consentement est précaire, voire impossible (mineurs, majeurs sous tutelle, personnes séjournant dans un établissement sanitaire ou social, malades en situation d'urgence).

Il peut exister en recherche comportementale des cas d'investigation "avec bénéfice individuel direct", par exemple en psychologie clinique lors d'une étude comparative de deux méthodes psychothérapeutiques (si pour les sujets qui s'y prêtent le bénéfice d'au moins l'une des méthodes est établi), ou en psychologie cognitive (si le fait pour des enfants de se prêter à une étude leur donne un avantage éducatif). Mais l'absence d'urgences vitales obligeant le praticien à intervenir, et la difficulté d'argumenter qu'une recherche est conduite "pour le bien" des sujets inclus, ou "dans l'intérêt de leur santé ", fait que la 
distinction "avec/sans bénéfice individuel direct" n'est pas pertinente en recherche comportementale pour relâcher les conditions du consentement, même quand il s'agit de recherche appliquée. Ia distinction n'est pertinente que dans le cadre du bilan risques/avantages.

Le C.CNE notait dans son rapport de 1990) qu'en règle générale les personnes qui se prêtent à une investigation dont l'objectif est l'acquisition d'une commaissance sur l'être humain n'y trouvent pas d'avantage persomnel. Le C.CNE a considéré que cela est admissible "à la condition d'un bilan risques/avantages acceptable, c'est-à-dire avantage certain pour la communauté, risque nul ou minime pour l'individu» $(p .70)$.

Faire le bilan risques/avantages, c'est se demander si les risques, contraintes ou désagréments imposés aux sujets ("coût humain") sont suffisamment justifiés par l'importance scientifique de la question posée, et par l'assurance que le protocole domne de résoudre cette question.

En recherche comportementale, outre qu'il convient de faire une évaluation explicite des risques physiques, on doit être particulièrement attentif aux risques psychologiques des expériences et à leurs conséquences possibles (pas d'expériences humiliantes, dégradantes ou traumatisantes).

\section{La justice. La dignité}

Dans la recherche biomédicale, le principe de justice sert d'abord à rappeler que la recherche scientifique ne doit pas être l'occasion d'une exploitation (par exemple, exploitation par des chercheurs de pays développés de populations pauvres de pays en développement, qui servent de " cobayes" pour l'acquisition de commaissances dont les retombées thérapeutiques bénéficient surtout aux populations des pays riches: (OMS-C.IOMS, 1982). Il sert ensuite à rappeler, comme le C.NE l'a fait dans son rapport de 1990 (p. 72), que la participation à un protocole de recherche appelle une "juste indemnisation" ou "compensation" des sujets, mais exclut toute rémunération.

En recherche comportementale, des cas d'exploitation (Nord-Sud) ont été éroqués (en ethnologie, en anthropologie), mais il y avait en général à l'arrière-plan un problème de discrimination ethnique ou culturelle (relation du développé au sauvage). Des risques francs de discrimination ont été évoqués à propos de l'investigation de traits distinctifs source de dévalorisation ou de dévalorisation sociale ("quotient intellectuel ", "chromosome du crime", etc.). En revanche, en recherche comportementale, la pratique de rémunérer les sujets (sur vacations, par exemple) n'a soulevé jusqu'ici que peu d'objections. Il existe des professionnels salariés (pilotes d'essai, plongeurs sous-marins) qui acceptent d'être des cobayes dans l'exercice de leur profession. Inversement, beaucoup de volontaires ne sont pas indemnisés (quand leur contribution est minime, ou que, comme dans le cas des étudiants, ils en tirent un bénéfice intellectuel ou didactique). Il n'est donc possible de dire, ni que tous les volontaires doivent être indemnisés, ni que toute rémunération est contraire à l'éthique.

Du point de v'ue de l'équité, le problème central en recherche comportementale semble donc être celui d'une possible discrimination, qu'elle soit liée au protocole de recherche lui-même, ou aux résultats de la recherche et à la manière dont ils seront compris et utilisés ( retombées" socioculturelles).

\section{L'examen éthique par des instances "indépendantes"}

Pour la recherche biomédicale, la loi française soumet à l'examen en C.C.PPRB les essais ou expérimentations. Elle exempte les "études" (travaux de recherche effectués sur dossier, ou sur des échantillons prélevés indépendamment de l'étude (exemple: sérothèque).

Les directives fédérales américaines (DHHS, 1981) prévoyaient trois catégories de recherche sur l'homme: exemptée de révision éthique (exempted research), soumise à une procédure de révision éthique simplifiée (expedited review, par le bureau d'un IRB), soumise à discussion éthique approfondie (discussion en séance par un IRB).

On pourrait proposer pour les sciences humaines une distinction entre simple observation et mise en situation expérimentale (?) pour prévoir une procédure rapide (simple examen du protocole), et une procédure normale d'examen éthique (pouvant requérir la présence effective des investigateurs). Cette distinction est toutefois d'un maniement délicat: il y a en effet des "expérimentations très anodines", et des observations éventuellement traumatisantes.

\section{Problèmes particuliers à la recherche comportementale, identifiés grâce aux protocoles soumis par le Cnrs}

\section{Consentement}

- Sous quelles conditions une recherche comportementale est-elle admissible sur des sujets dont l'aptitude à consentir est problématique (compétence limitée, dépendance) ? La règle générale est que les personnes dont le consentement est précaire doivent être protégées contre l'éventualité de servir de "sujets" de recherche, à proportion de leur incapacité et de leur dépendance. Quelques catégories vulnérables à protéger spécialement en recherche comportementale sont: enfants, infirmes mentaux, personnes aisément manipulables par une faiblesse ou une dépendance qui leur est propre (exemple: toxicomanes), populations captives institutionnalisées (détenus, élèves, adolescents relevant de l'éducation surveillée, jeunes en foyers, militaires).

- Pour les mineurs et majeurs sous tutelle, la règle admise pour la recherche biomédicale (L. 209-10) peut être généralisée : consentement des parents ou tuteurs légaux, infor- 
mation de l'enfant ou de l'incapable dans toute la mesure du possible, respect d'un éventuel refus de l'enfant ou de l'incapable.

- Pour les populations captives institutionnalisées (détenus, élèves, adolescents des "maisons de redressement ", jeunes en foyers), la règle habituelle est de ne juger admissibles que des recherches: (1) à "risque minime "; et (2) non discriminatoires. Le consentement doit être obtenu, d'une part, des responsables de l'institution, d'autre part, de chaque sujet individuellement (si les sujets sont mineurs, de la persomne qui détient l'autorité parentale).

- L'usage de "récompenses" doit faire l'objet d'une attention spéciale au moment de la révision éthique (exemple : recrutement de clochards ou d'indiens comme sujets de recherche par une offre d'alcool). - En ce qui concerne les détenus, la loi du 20 décembre 1988 exclut les recherches sans bénéfice individuel direct chez les persomnes privées de liberté. Dans les sciences du comportement, ces recherches peuvent être sans nocivité pour les personnes concernées, et socialement utiles, voire nécessaires; elles ne peuvent donc être prohibées sans autre examen.

- Comment concilier l'obligation d'un consentement qui devrait être éclairé avec la nécessité méthodologique (qui peut se présenter dans certains protocoles expérimentaux) de ne pas tout dire? Il s'agit d'une question évoquée à propos de la recherche biomédicale, mais surtout connue à propos de la psychologie, de la psychologie sociale ${ }^{(t)}$, et de la recherche sur les performances sportives.

Le Conseil de recherches médicales (CDR) canadien a traité cette question comme suit :

F. Tromperie (5)

“... Vu l'importance critique du consentement libre et éclairé, il sem-

(4) Voir expériences de Stanler Milgram, éooquées dans le film 'I'comme Icare. Pablication de regé rence: Milgram.S. Behanioral sludy of obedience. J Abnorm Pswchol 1963; 67: 37I-8. le incongru de se pencher sur la question de la tromperie.

On entend par tromperie le fait d'induire volontairement en erreur les sujets éventuels ou de leur cacher des renseignements, de façon à les amener à croire que les objectifs de la recherche ou de la façon de procéder sont différents de ce qu'ils sont en réalité. I a tromperie peut également consister à leur présenter délibérément de faux renseignements, à dissimuler des renseignements importants, ou à ne révéler que des bribes d'information, de façon à induire les intéressés en erreur.

La tromperie étant à ce point contraire au principe du respect de l'individu, le Comité a eu énormément de difficulté à admettre l'idée qu'elle puisse parfois être justifiable d'un point de vue éthique. Il reste que si, pour une raison ou une autre, il était indispensable que les sujets ne soient pas mis au courant de la nature des recherches, et ce, pour l'intégrité scientifique, il faudrait s'assurer que les règles suivantes sont observées: (1) on ne doit jamais avoir recours à la tromperie lorsqu'il existe un autre moyen d'atteindre les objectifs de la recherche. Le chercheur doit montrer, dans le protocole, qu'il n'y a aucune autre façon de procéder; (2) on doit s'abstenir de mener des recherches de caractère trompeur lorsqu'il pourrait y avoir des risques pour les sujets: imposer des risques à un sujet qui n'a pas accordé son consentement est impossible à justifier ; (3) le C.DR doit avoir l'assurance que l'on ne dissimulera aucun élément d'information qui, s'il était révélé, entraînerait un refus de participer; (4) le C.DR doit avoir l'assurance que les recherches pourraient aboutir à des progrès scientifiques considérables, pour justifier le recours à la moindre duperie; (5) la tromperie ne doit être admise que lorsqu'il est possible d'informer les sujets et de leur faire un compte rendu de l'expérience une fois la recherche terminée, et d'obtenir leur consentement pour l'utiịisation des domnées recueillies.
La méthode de compte rendu doit être indiquée dans le protocole de recherche et cette démarche doit avoir lieu immédiatement après la participation aux recherches, lorsque les domnées permettent encore d'identifier les sujets. Il ne faut pas perdre de vue, toutefois, qu'elle n'anmule pas pour autant la tromperie. Les données concernant les sujets qui refusent de domner leur consentement à l'étude doivent être détruites ou restituées aux intéressés. Cette condition aura, à notre avis, un effet dissuasif sur le recours à la tromperie." (CRM, 1986, A, C.h. 5, \$).

On peut proposer les règles suivantes.

1. Le protocole doit apporter des arguments prouvant que l'acquisition de la comnaissance cherchée présente un intérêt scientifique, que la dissimulation de certains aspects du protocole est indispensable pour atteindre l'objectif visé, et qu'aucun des aspects dissimulés aux sujets n'est susceptible de menacer leur sécurité ou leur dignité, ou de les dissuader de consentir s'ils leur étaient révélés.

2. Le ('CPPRC' doit avoir admis ces arguments, jugé que la situation dans laquelle les sujets de l'étude seront placés est acceptable, et avoir domné un avis favorable à l'étude. 3. Les sujets sont informés au moment du recueil initial de leur consentement que certains aspects de la méthodologie leur sont délibérément cachés, que cela est nécessaire à l'étude, que c'est "la règle du jeu ", que le C.C.PPRC: a jugé que rien de ce qu'on leur dissimule ne les menace, ni dans leur sécurité, ni dans leur dignité, et qu'à la fin de l'étude on les éclairera et répondra à toutes leurs questions.

4. A la fin de l'étude, les sujets, s'ils le souhaitent, sont mis complètement au courant de l'objet des recherches et des observations faites sur eux-mêmes ${ }^{(i)}$, et il est répondu à toutes leurs questions. Ils sont informés de l'usage qui sera fait des domnées recueillies. Au cas où des

(6) En anglais: debriefing 
données identifiantes (photos, films) ont été recueillies, les persomnes concernées doivent domner un consentement explicite à leur utilisation. L.es données identifiantes concernant des persommes qui refusent de consentir à l'utilisation qu'on veut en faire seront détruites ou restituées aux intéressés.

\section{Sécurité}

Comment évaluer les risques psychologiques, et les séquelles éventuelles pour les sujets, d'une recherche comportementale?

Il a été argumenté à propos de la recherche biomédicale que, par définition, quand on cherche, les risques sont inconnus, et par conséquent ne sont pas évaluables. A cet argument on oppose habituellement le contre-argument que les hypothèses sur lesquelles s'appuie une recherche sont fondées sur des connaissances déjà acquises, et que ces connaissances d'arrière-plan permettent une appréciation au moins approximative des risques encourus par les sujets soumis à l'expérience. Le même raisonnement vaut pour la psychologie.

(Rappel : techniquement, un risque est le produit d'une gravité par une probabilité. L'évaluation du risque comporte donc une double appréciation: de la gravité des troubles susceptibles d'être provoqués che\% le sujet d'expérience, de la probabilité d'occurrence de ces troubles.)

La distinction entre "risque minimal" (= négligeable) et "risque non minimal" (= non négligeable, sérieux $\left.{ }^{(7)}\right)$ a été introduite en 1978 par la Commission nationale américaine. Le "risque nul" n'existe pas

\footnotetext{
(7) La loi frangaise du 20 décembre 1988 dit "risque sévieux", el slipule que pour les personnes appartenant à des catégories zulnérables la recherche "sans bénéfice individuel dired" n'est admissible que si elle ne présente "aucun risque sérieux prévisible" (.Art. I. 2()9-6).

(8) le Rappont alu Royal College of Physicians of I.ondon (1986) montre que le ristue est jugé minimal, soil parce que le trouble encoume est d'une très faible grazrilé. soil parse que la probabilité d'accident est tròs faible. Dans ce dermier cas, le risque "est comparable an risque encomm par un $m / s n^{\circ} 12$ zol. 9. desemlne 93
}

dans les entreprises humaines. Les risques minimaux sont des risques du même ordre de grandeur que les risques pris couramment sans y penser dans la vie quotidiemne. Ainsi, pour les enfants, le risque minimal est défini comme celui dont "la probabilité et la gravité sont du même ordre que celles des dommages physiques ou psychologiques auxquels les enfants en bonne santé sont normalement exposés dans leur vie, ou dans des examens médicaux ou psychologiques de routine" (National Commission ..., 1978-12, App. 1) ${ }^{(8)}$.

Exemples de risques sérieux: expériences d'isolement hors du temps, de privation sensorielle, d'endurance à des conditions extrêmes, etc.

Aux catégories de sujets vulnérables il ne peut être proposé que des expériences dont les risques sont d'un niveau minimal, ou, pour reprendre les termes de la loi française de 1988, des expériences qui ne présentent aucun risque sérieux prévisible.

C.e n'est qu'à des sujets en bonne santé, adultes, en pleine possession de leurs facultés mentales, et pleinement informés, qu'on peut proposer de se prêter pour les besoins de la recherche en sciences humaines à des expériences comportant des risques d'un niveau supérieur (aussi peu que ce soit) au niveau minimal. - Comment concilier l'impératif de confidentialité des domnées avec la communication à des chercheurs non médecins, en vue de recherche, de dossiers médicaux confidentiels? Le problème d'éthique est un problème de respect de la sphère persomnelle, et de non-divulgation de domnées confidentielles dont le médecin traitant est dépositaire. On peut se fier aux articles 7 à 13 du Code de déontologie des psychologues (note 2), calqués sur les règles du secret médical.

Au-delà du problème d'éthique, il y a en France un problème légal et réglementaire. Quelques textes de référence sont: Art. 226.13 et 226.14 (ancien Art. 378) du Code pénal ${ }^{(9)}$, Loi $n^{\circ} 78-17$ du 6 janvier 1978 relative i l'informatique, aux fichiers el aux libertés, Décret n 79-506 du 28 juin 1979 portant Code de déontologie médicale. Ces textes excluent la communication par le médecin de données nominatives à quiconque, sauf à un autre médecin et dans l'intérêt strict de la santé d'un malade (exemple: consultation d'un spécialiste pour un avis thérapeutique).

Le problème de la communication de domnées en vue de la recherche a été étudié de façon approfondie dans les années 1980 à propos des registres du cancer et de la recherche épidémiologique, entre la CNIL, le C.CNE, l'Ordre des médecins. La solution proposée avait été celle du secret professionnel partagé. Cette solution n'est pas encore légale (10). En l'état actuel de la législation, seule est admissible (dans le cadre hospitalier, par exemple) une étude utilisant des domnées médicales nominatives à l'intérieur du service où les patients ont été traités, et sous la responsabilité d'un médecin du service. C'est le médecin responsable du service (et non le chercheur psychologue) qui doit prendre contact avec les familles, et solliciter leur consentement à la recherche. Les familles pourraient passager d'azion utilisant un zol régnalier" (voir Rapport Menard, 199())

(9) Art. 226.13: "In révélalion diune in fommation ì caraclère secrel par une personme qui en est déposilaire, soil par étal ou par profession, soil en raison d'ume fonclion ou d'une mission lemponraire, est punie d'un an d'emprisommement el de 10()()()()$F$ diamende."Arl. 226.14: "L'article 226.13 n'est pas applicable dams les cas où la loi impose on aulo rise la révélation du secrel. En outre, il n'est pas apt)licable: (l) A celui qui informe les aulorilés judiciaires, médicales ou administratives de sévices ou privalions dont il a eu connaissance ef quii ont été infligés ì un mineur de quinze ans ou ì une personne qui n'est pas en mesure de se prolegger en raisom de son âge ou de son élal physique ou psychique; (2) au médecin qui avec l'accord de la viclime porle a la connaissance du Procureur de la République les sévices qu'il a constalés dans l'exerrice de sa profession el qui lui permellent de présumer que des ziolences sexuelles de lonte nalure ont élé $\mathrm{com}-$ mises. "Loi $n^{\circ} 92.684$ du 22 juillel 1992, pame au Journal officiel du 23 juillet 1992, portant reforme du Cisde pénal. (ielle loi entre en riggueur lo ()1-()3-94. 
légitimement se plaindre qu'un psychologue les ait contactées (par téléphone, puis directement) pour une recherche sur leurs jumeaux, ce qui impliquerait que ce psychologue ait pris connaissance de leur dossier médical avant tout consentement de leur part, et donc que le chef de service concerné n'ait pas respecté le secret médical.

L'utilisation de données médicales nominatives en vue de recherche est donc obligatoirement sous la responsabilité du médecin, les personnes concernées ayant le droit d'être informées préalablement de l'usage qu'on veut faire de leurs domnées, et le droit de s'y opposer. On pourrait imaginer que des chercheurs psychologues (ou statisticiens, ou biologistes, ou anthropologues ...) (non médecins) soient désormais habilités à prendre connaissance de certaines données médicales nominatives en vue d'une recherche, et à les traiter sous leur propre responsabilité. Par mesure d'équité, cela impliquerait qu'inversement des médecins puissent être habilités à prendre comnaissance, en vue de recherche, de données psychologiques nominatives.

Cela supposerait: (1) une procédure d'habilitation, (2) une modification des textes législatifs en vue de rendre admissible la notion de secret professionnel partagé entre médecins et non-médecins.

Notons que la loi «informatique et libertés" du 6 janvier 1978 n'autorise à déroger au secret médical au bénéfice de la recherche qu'à la condition que les personnes concernées aient domné leur accord. Or le Code de déontologie médicale français ne prévoit pas que le patient puisse délier le médecin du secret (les seuls cas de dérogation au secret sont ceux prescrits par la loi) (voir Villey, 1986, p. 135). Le Code de déontologie des psychologues va plus loin: "En dehors des cas d'obligation légale, le psychologue ne peut être délié de son secret par quiconque, même par ceux que ce secret concerne" (Art. 13). La justification de la règle que même la personne concernée ne peut délier le médecin ou le psychologue du secret est que, pour délier quelqu'un du secret, il faut savoir ce qu'il est. Or le psychologue clinique n'est pas tenu de communiquer ses fiches à son client. Et le malade français n'a qu'un accès indirect (111) à son dossier médical, dont il ignore généralement ce qu'il contient.

Si la législation française s'oriente vers une nouvelle dérogation au secret médical, en vue de recherche scientifique, cela implique donc aussi une modification du Code de déontologie médicale, une modification du Code de déontologie des psychologues, et sans doute à terme pour tous les malades un droit d'accès direct à leur dossier médical, donc une modification de la loi informatique et libertés, et de la loi sur l'accès aux documents administratifs.

\section{Problèmes d'équité}

- Comment concilier la recherche sur des traits discriminants avec l'impératif de non-discrimination?

Il s'agit d'un problème délicat, parce que l'identification de différences significatives et de traits discriminants est précisément un objectif plausible de recherche. Ainsi, il est intéressant, et important pour l'évaluation des techniques de pro- création médicalement assistée, de se demander s'il existe une différence significative entre le développement cognitif des enfants nés d'une insémination artificielle avec domneur et les autres. Mais si l'on prouve que les enfants nés par insémination artificielle avec donneur se développent moins bien que les autres, et que le résultat de la recherche est rendu public, les enfants déjà nés dans ces conditions (ou leurs parents) sont mis en position difficile, même si le secret des origines est bien gardé (danger de culpabilisation).

Il faut que toutes les précautions soient prises pour que la recherche ne soit pas source de discrimination par sa méthodologie. Outre la méthodologie, une recherche peut comporter un risque de discrimination explicite ou implicite contre des individus par les hypothèses sur lesquelles elle repose (exemple : expérimentation de "thérapies d'aversion" pour soigner les homosexuels: suivi psychosocial d'une cohorte d'enfants nés de mères alcooliques). Une recherche peut aussi entrainer un risque de discrimination contre toute une population par l'interprétation qui sera faite de ses résultats (exemple: mise en évidence de résultats statistiquement moins bons à quelques épreuves de tests d'intelligence chez les Noirs que che\% les Blancs).

Aux Etats-Unis, à la fin des années 1960, le souci d'une science for the people fit stopper des investigations, au motif que leurs implications théoriques étaient infamantes pour certaines catégories d'individus. On cite même des tentatives pour contraindre les chercheurs à "réviser" leurs conclusions et/ou ne

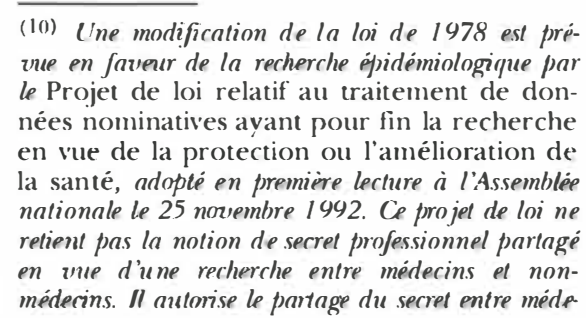

cins en vue d'une recherche. Ie cas de la recherche en prychologie n'est pas pris en consideration par le projet de loi qui vise essentiellement le traitement statistique de données nominatives pour des objectifs de recherche en santé publique. In transmission de données à l'extérienur d'un service hospitalier vers une institution de recherche on zue d'une investigation (exemple: traitement statistique) est automsée par le projel de loi i la condition que le futur Comité consultatif national sur le traitement de l'information en matière de recherche en santé ait donné son accord a l'élude, que les personnes concernées aient été informées individuellement et aient pu exercer lour droit d'opposition (sauf impossibilité), que les données soient réceptionnées par un médecin désigné par l'organisme de recherche et chargé de veiller à la sécurité de ces données et au respect des finalités de la recherche.

(11) P'ur l'intermédiaive d'un médecin de son choix. 
publier que des résultats expurgés. Les risques de discrimination sont fonction du niveau de tolérance sociale à telle ou telle "différence ". On peut proposer la règle: les risques de discrimination doivent être identifiés, et mis en balance avec l'intérêt collectif et l'intérêt théorique d'obtenir le résultat cherché.

- Faut-il que les promoteurs d'une recherche comportementale souscrivent une assurance couvrant les risques encourus par les sujets de recherche du fait de l'investigation ? En recherche biomédicale, la loi française fait obligation au promoteur de souscrire une assurance (Art. L. 209-7) garantissant sa responsabilité civile en cas de conséquences dommageables de la recherche pour la personne qui s'y prête. On sait que cette disposition de la loi ne pose aucun problème quand le promoteur est l'industrie pharmaceutique, mais qu'elle pose problème pour des recherches conduites par des équipes universitaires et/ou dans des hoppitaux de taille modeste, qui ont du mal à trouver un promoteur ayant une assise financière suffisante pour souscrire l'assurance.

En recherche comportementale, les grands organismes de recherche (Cinrs, laboratoires universitaires) sont-ils aptes à se porter promoteurs de recherche? Faute d'identifier le promoteur, on ne peut, en effet, poser le problème de l'assurance de façon satisfaisante.

On pourrait argumenter qu'en cas de recherche comportementale à risque minimal, l'assurance n'est pas indispensable, encore qu'il y ait des risques physiques à prendre en compte, qui, sans être une conséquence directe de l'acte de recherche, se produisent à l'occasion de la recherche (chutes, accidents de trajets). On pourrait aussi argumenter que, pour les recherches à risque plus élevé, comme elles sont proposées seulement à des volontaires majeurs et responsables, pleinement consentants, il incombe à ces volontaires de souscrire une assurance, comme pour d'autres activités à risque (exemple: activités sportives). Mais en cas de litige, il pourra tou$\mathrm{m} / \mathrm{s} n^{\circ} \quad 12$ nol. 9, decembre 93 jours être argumenté que les chercheurs responsables du projet n'auraient pas dû proposer aux volontaires une telle situation à risque. Et il existe à notre connaissance en France au moins un exemple de suicide consécutif à une recherche comportementale à risque (isolement prolongé "hors du temps").

Le problème de l'assurance pour les recherches comportementales sur l'homme doit donc au moins être posé. Le résoudre pour la généralité des cas est l'affaire du législateur.

Examen des protocoles. Que seraient les CCPPRC (Comités consultatifs de protection des personnes dans la recherche comportementale)?

Il paraît exclu que les C.C.PPRB dans leur composition actuelle puissent examiner les protocoles de recherche en sciences humaines. Ils n'ont pas été conçus pour cela.

Il n'entre pas dans les missions de la section technique du C.C.NE de procéder à l'examen systématique de protocoles de recherche en sciences humaines.

L'expérience française antérieure à la loi $n^{\circ} 88-1138$ du 20 décembre 1988 (exemple AP de Paris), ainsi que le modèle nord-américain, iraient dans le sens de la création de Comités d'éthique de la recherche en sciences humaines auprès d'institutions qui, de fait, font de la recherche comportementale sur des sujets humains: (inrs, universités. Selon R.J. Levine (1986), ces comités sont «la conscience de l'institution", et l'expression de son souci d'assurer en son sein une recherche de qualité, et une protection adéquate des persomnes qui s'y prêtent. Des règles touchant la composition de ces comités (qui devraient inclure une certaine proportion de membres extérieurs à l'institution, et/ou extérieurs aux sciences humaines: juristes, philosophes, médecins) pourraient être élaborées par une commission mixte (Conrs-universités-C.CNE-ministères-organisations professionnelles). Une procédure d'habilitation de ces comités pour- rait être définie par un texte du Ministère de la Recherche. Ce serait probablement une solution provisoire, mais qui pourrait être mise en place assez vite.

Dans le contexte français, il semble difficile de ne pas envisager à plus long terme la mise en place par la loi de quelques C.C.PPRC: régionaux, sur le modèle des C.C.PPRB, mais ayant une composition qui leur assure la compétence requise pour examiner des protocoles de recherche sur l'être humain dans les sciences humaines autres que biomédicales: anthropologie, ethnologie, psychologie(s), sociologie, linguistique, histoire, sciences de l'éducation, etc. ('’est l'affaire du législateur.

Le dossier soumis à un C.CPPRC: devrait fournir au minimum les informations suivantes: (1) les objectifs de la recherche et les hypothèses de travail, (2) la population visée par l'étude, et les modes de recrutement des sujets (y compris le texte publicitaire éventuel), (3) le détail de la méthodologie, (4) les dispositions prises pour anonymer les domnées recueillies, les conserver, et en permettre la consultation, (5) l'évaluation des risques, des coûts, des contraintes et astreintes, des bénéfices potentiels, (6) l'information des personnes participant à l'étude et les modalités de leur consentement, y compris le document de présentation de l'étude destiné à ces personnes, et le formulaire de consentement (consentement à l'étude, et, le cas échéant, à l'utilisation des résultats)

\section{BIBLIOGRAPHIE}

American Psychological Association. Ethical issues in psuchological research in AIDS, Committee for the protection of human participants in research. IRB 1986; $8(4): 8-10$.

Barber B. The ethics of experimentation with human subjects. Scientific American $1976: 234: 25-31$.

Baumrind I). IRBs and social science research : the costs of deception. IRB 1979; 1 (6) : $1-4$. 
Bourdieu P, ed. I.a misère du monde. Paris: Seuil, 1993.

Conseil de recherches médicales du Canada. Lignes directrices concemant la recherche sur des sujets humains, Ottawa, 1986.

Cupples B, Gochnauer Myrom. The investigator's duty not to deceive. IRB 1985; 7 (5) : $1-6$

de Sola Pool I. Do social scientists have unlimited research rights? IRB 1983;5 (6) : 10 .

Desportes JP. I .es manipulations du comportement. I. Recherche 1974; 47 : 654-61.

Fagot-largeault A. L.'homme bio-élhique. Poner une déontologie de la recherche sur le vivant. Paris: Maloine, 1985.

Gordis I., Gold E. Priwacy, confidentiality, and the use of medical records in research. Science 1980); 207: 153-6.

Gosselin C. Une Eilhique des Sciences Sociales. Paris: L.'Harmattan, 1992.

Harris SL, et al. Behavior modification therapy with elderly demented patients: implementation and ethical considerations. J (hronic Dis 1977: 30 : 129-34.

Kat/ J. Experimentation wilh Human Beings? The Authority of the Investigator. Subject, Pro Jessions and State in the Human Experimentation Process. New York: Russell Sage Foumdation, 1972.

Levine C. Former soldier denied compensation for damage in army I.SD tests. IRI $1982: 4(3): 7$.

Levine RJ. Eilhics and Regulation of Clinical Research, $2^{\text {nd }}$ ed. Baltimore-Munich : Urban and Schwarzenberg, 1986.

Macklin R. The paradoxical case of payment as benefit to research subjects. $I / R B$ $1989: 11(6): 1-3$

Marini Jl. Methodology and ethics: research on human agression. IRB 198(): $2(5): 1-4$

Mead M. Research wih human beings : a model derived from anthropological field practice. Docedalus 1969:98: 361-86.

Meyer RE. Subjects' rights, freedom of inquiry, and the future of research in the addictions. Am I Psochialr 1977: 134: $899-9() 3$

Menard J. Rapport du groupe de réflexion Inserm sur certains aspects de la protection des sujets volonaires sains et des persomnes qui se prêtent à des recherches biomédicales. Paris: Inserm, 199().
Human Subjects of Biomedical and Behavioral Research. Research Imolving Risomers: Report and Recommendalions, and Appendix (1976). Research Involving (ihildren: Report and Recommendations, and dppendix (1977). Resparch Involving Those Institutionalized as Mentally Infinn: Report and Recommendations, and Appendix (1978). The Belmont Report: Eilhical Principles and (iuidelines for Research Involuing Human Suljects (1978). W'ashington DC: US (iovt Printing ( )ffice (DHEW) $\mathrm{Tr}$. fr. in: Médecine et expérimentation. Cahiers de bioéthique, 4. Québec: Presses de l'Université Laval, 1982: 233-50.

Newton I.H. Dentists and pseudo-patients: further meditations on deception in research. $I R B \quad 1982: 4(8): 6-8$.

Park I.C, Covi I, Lhlenhuth E.H. Effects of informed consent on research patients and study results. J Verv Ment Dis 1967: $145: 349-57$

Pattulo E.L. Who risks what in social research ? IRB 1980):2 (3): $1-3,12$.

Queheillard Jl. Secret professiomel: le grand oublié? Psychologues et psychologies 199()$, 11^{\circ} 89$.

Redlich F. The anthropologist as observer ethical aspects of clinical observations of behatior. / Vert Ment IDis 1973: 157 : 313-9.

Robertson JA. Fthical review of social experiments. IRB 1981:3 (7): 1()-1.

Rowal college of Phrsicians. Research on healthw volumteers. JR (oll Phssicians Lond, $1986: 20: 243-57$

Schafer A. The ethics of research on human beings: a critical review of the issues and arguments. Res ddv Alrohol Drng Probl 1981: 6: 471-511.

Schiff M. L.es impasses de la recherche en psychologie. Psychologues el psychologies 1991 $10^{\circ} 1() 4$.

Schmutte (iT. Using students as subjects without their knowledge. IR/ 1980): (10): $5-6$

Schuler H. Eilhische Probleme psychologischer liorschung. (ï̈ttingen.

Sieber JE. How humanism and determinism differ: understanding risk in psvchor logical research. IRB 1982: 4 (3) : 1-3, 12

Sieber JE. Deception in social research. I: hinds of deception and the wrong they may involve. IRB 1982; 4 (9) : 1-5.

Sieber JE. Deception in social research. II : Evaluating the potential for harm or wrong. IRR 1983: 5 (1) : 1-6.

Sieber JE. Deception in social research. III The nature and limits of debriefing. IRI $1983: 5(3): 1-4$
Sieber JE. (On studving the powerful (or fearing to do so) : a vital role for IRBs. IRIB $1989: 11(5): 1-6$.

St James-Roberts 1 . Are researchers trustworthy: New Scientist 1976: 171: 481-3.

Thouvenin D. I.influence de la loi $11^{\circ} 88-1138$ du 20 décembre 1988 (modifiće $\left.11^{\circ} 9()-86\right)$ sur l'organisation de la recherche. (iestions hospitalieres - la recherche L.hopital 1992; $11^{\circ} .320$.

Thouvenin D). (onsentement et assujettissement. In: Gros and Huber, eds. Vers un anti-destin? Palrimoine génélique el droil de Thumanilé. Paris: Editions (Odile Jacob, 1992: 471-8.

U'S Departement of Health and Human Services. Final regulations amending basic HHS policy for the protection of human research subjects, 26 janvier 1981 . Fed Reg $1981: 46$ (16): $8366(-92$.

Villev R. Histoire du secrel médical. Paris: Seghers, 1986.

American Anthropological Association, code of ethics.

Anthropologie el sociélés, du Département d'anthropologie de I aral, Québec, numéro spécial "comprendre et modifier", 1984. vol. $8,1^{\circ} 3$, incluant un code de déontor logie prof essiomelle.

Code d'éthique de l'Association brésilieme d'anthropologie, adopté lors de sa 16" réunion, à (ampinas (Sao Paulo), 30) mars 1988 ("Codico de Etica").

Ciurrem Anthropology. (Chicago, vol IX, 5, 1968, puis vol $\mathrm{xl}, 1,1970$, enfin vol. Xil, 1 , 1971 (a partir d'un sumposium "on the social responsibilities in social anthropor logn'”.

Journal des anthropologues. Hiver 92-printemps 93, $11^{\circ} 5(0)-511$, consacré à "l'éthique professiomnelle" et aux "expériences de terrain" (E.HESS, 1, rue du I1-novembre, 92120 Montrouge).

Sociélés comtemporaines (IRES(C), C.urs), numéro spécial "E.thique professionnelle" septembre 1991, $1^{\circ} 7$ (érhique des statisticiens, des généticiens, en anthropologie, etc.)

Sociolog; novembre 1992, "BSA Statement of Ethical Practice" 